\title{
Prediction of fatigue life of aluminum 2024-T3 at low temperature by finite element analysis
}

\begin{abstract}
The change in material properties at low temperature has always been one of the concerned design factors in aircraft industries. The wings and fuselage are repeatedly exposed to sub zero temperature during cruising at high altitude. In this study, fatigue tests were conducted on standard flat specimens of aluminum 2024-T3 at room temperature and at $-30{ }^{\circ} \mathrm{C}$. The monotonic and cyclic loading tests were conducted using MTS 810 servo hydraulic machine equipped with a cooling chamber. The monotonic tests were conducted at a crosshead speed rate of $1 \mathrm{~mm} / \mathrm{min}$ and the cyclic tests at a frequency of $10 \mathrm{~Hz}$ with a load ratio of 0.1 . The experimental data obtained, such as the yield strength, ultimate strength and S-N curve were used as the input parameters in ANSYS Workbench 16.1. This close agreement demonstrates that the isotropic model in ANSYS workbench is essential in predicting fatigue life. The increase in stress parameter causes fatigue life to decrease. Besides, the decrease in temperature causes the total fatigue life to increase.
\end{abstract}

Keyword: Aluminum 2024-T3; Fatigue test; Low temperature; ANSYS Workbench 